\title{
Precise calculations of the deuteron quadrupole moment
}

\author{
Franz Gross** \\ Jefferson Lab, Newport News, VA, USA \& \\ College of William and Mary, Williamsburg, VA, USA \\ E-mail: grossejlab.org
}

\begin{abstract}
Recently, two calculations of the deuteron quadrupole moment have have given predictions that agree with the measured value to within $1 \%$, resolving a long-standing discrepancy. One of these uses the covariant spectator theory (CST) and the other chiral effective field theory $(\chi \mathrm{EFT})$. In this talk I will first briefly review the foundations and history of the CST, and then compare these two calculations with emphasis on how the same physical processes are being described using very different language. The comparison of the two methods gives new insights into the dynamics of the low energy NN interaction.
\end{abstract}

The 8th International Workshop on Chiral Dynamics

29 June 2015 - 03 July 2015

Pisa, Italy

\footnotetext{
* Speaker.

$\dagger$ This material is based in part on work supported by the by Jefferson Science Associates, LLC, under U.S. DOE Contract No. DE-AC05-06OR23177.
} 


\section{History and Overview}

Some recent calculations of the deuteron quadrupole moment, $Q_{d}$, are summarized in Table 1. All of these calculations use realistic NN scattering models with kernels or potentials adjusted to fit the low energy $\mathrm{NN}$ data to high precision, tightly constraining the calculations of $Q_{d}$. As the table shows, earlier calculations consistently under-predicted its value by several percent $[1,2,3$, 4], leading Machleidt [3] to conclude that this failure was an "unresolved problem." The recent calculations using $\chi$ EFT (with cutoffs somewhat below $600 \mathrm{MeV}$ ) and the CST (model WJC-2) obtain results that agree with experiment to within $1 \%$, resolving this problem and adding to the successes of meson theory.

\begin{tabular}{lll}
\hline year: reference & \multicolumn{1}{c}{$\delta Q_{\text {pred }}=\left(Q_{\text {pred }}-Q_{\exp }\right) / Q_{\exp }$ (model) } \\
\hline 92: Early CST (VanOrden, et.al. [1]) & $-9.0 \%$ (IIB) & $-8.1 \%$ (IIB with RC) \\
95: Argonne (Wiringa, Stoks, Schiavilla [2] $)$ & & $-3.8 \%$ (with MEC) \\
01: CD Bonn (Machleidt [3]) & $-5.6 \%$ (no MEC) & $-2.1 \%$ (MEC est.) \\
09: Light-Front (Huang \& Polyzou [4]) & $-5.7 \%$ (IM) & $-3.8 \%$ (IM+Ex) \\
13: $\chi$ EFT, ODU-Pisa (Piarulli, et.al. [5]) & $-0.3 \%$ (500) & $-1.4 \%$ (600) \\
15: Full CST(Gross [6]) & $-2.5 \%$ (WJC-1) & $-0.8 \%$ (WJC-2) \\
\hline
\end{tabular}

Table 1: Predictions for the quadrupole moment, expressed as a percentage "error" with respect to the experimental value of $0.2859(6) \mathrm{fm}^{2}$.

In this talk I will first review the CST, and then compare the different languages used by $\chi$ EFT and the CST. This comparison grew, in part, out of recent work on a review of the few-body electromagnetic form factors [7]. The last two sections present a brief summary of the origin of the CST contributions and some final discussion.

\section{Review of the Covariant Spectator Theory}

\subsection{Overview}

The history of the CST can be very briefly summarized:

- 1969 (birth of the CST): Discovery that the generalized ladder sum (sum of ladders and all crossed ladders) for the case of two particles of equal mass $M$ is well approximated by the sum of ladders (only) with the particle on its positive energy mass-shell (defined to be the residue of the $k_{0}$ energy integral at the pole $k_{0}=\sqrt{M^{2}+\mathbf{k}^{2}}$ ) [8]. It was subsequently proved that, for scalar particles with unequal masses $M>m$, the result is exact in the limit $M \rightarrow \infty$ $[9,10]$. This is referred to as the cancellation theorem.

- 1987 (two-body currents, FG and D. O. Riska): Derivation of a two-body current operator that is exactly conserved, even in the presence of phenomenological strong form factors [11]. Since meson theories are always regulated by strong form factors, this opened the way to using the CST for precise studies of electromagnetic interactions. 
- 1992 (first OBE models, FG, J. W. Van Orden and K. Holinde): Application of the CST to NN scattering was developed and several OBE (one boson exchange) models were found that fit the low energy scattering data [1]. The $\chi^{2} /$ datum of these early models was around 3 to 4 .

- 1995 (deuteron form factors, J. W. Van Orden, N. Devine and FG): Deuteron form factors were calculated [12] using the currents from Ref. [11] and the relativistic wave functions from Ref. [1].

- 1997 (prediction of the ${ }^{3} \mathrm{H}$ bound state energy, A. Stadler and FG): Discovery that a CST OBE model can predict the three body binding energy of the triton without any three-body forces, provided the OBE couplings to the scalar mesons have off-shell terms [13].

- 1997: (three-body currents, Kvinikhidze and Blankleider) Using the method of "gauging of equations" a conserved three body current is derived [14]. Later, in 2003 with T. Peña and A. Stadler [15], these equations were reorganized into a practical form for calculation of three-body form factors, calculated by Pinto and Stadler in 2009 [16, 17]

- 2008 (precision OBE models, FG and A. Stadler): Development of the OBE model WJC-2 that gave a precise fit to the $\mathrm{np}$ scattering data below $350 \mathrm{MeV}\left(\chi^{2} /\right.$ datum $\left.\sim 1.1\right)$ using the exchange of the photon and only six bosons: $\pi, \eta, \sigma$ (scalar-isoscalar), $\delta$ (scalar-isovector), $\omega$, and $\rho$, and requiring only 15 adjustable parameters [18]. (Another model, WJC-1, gave a slightly better fit but required 27 parameters and used axial vector mesons as well. This model seems to be less favored because of its less accurate prediction of the quadrupole moment., but a final evaluation awaits new calculations of the deuteron form factors, currently underway.)

- 2014-2015 (deuteron form factors with new exchange currents): A unique two-body isoscalar interation current has been derived recently [19], and was used to calculate the magnetic moment [20], the quadrupole moment (reported here) [6], and is currently being used to calculate the deuteron form factors, expected to be completed in a few months.

I will complete this overview by emphasizing two features of the modern CST OBE models of the NN interaction: (i) there are no three (or many) body forces, and (ii) a successful CST OBE model must include off-shell couplings at the $\sigma N N$ vertices. The next two subsections explain these points more fully.

\subsection{CST OBE models do not have three-body forces}

The absence of three body forces in a CST OBE model is illustrated in Fig. 1 showing a subset of diagrams, up to sixth-order in the $b N N$ coupling constant, where particles 2 and 3 are the "last" to interact, leaving particle 1 as a spectator to this last interaction. As required by the CST expansion [13], the internal propagation of the three nucleons between each meson exchange has only one nucleon off-shell. It is therefore easy to see, using the three-body CST equation, that these diagrams (and, by extension, all such OBE diagrams) can be generated by iterating elementary diagrams with an OBE exchange between two of the three nucleons, with the third nucleon a spectator. No three-body forces are needed to generate any of these diagrams. 


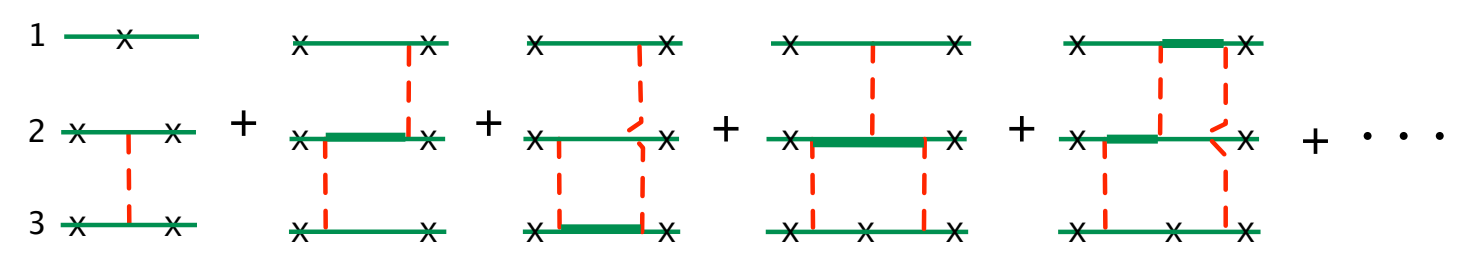

Figure 1: Diagramatic representation of the CST OBE Feynman diagrams for three-body scattering, with on-shell particles labeled by an $\times$. In these diagrams, the sequence of interactions is from right to left, but they are not time ordered.

\subsection{Successful CST OBE models include off-shell couplings}

Since the CST allows one nucleon to be off-shell, off-shell couplings at the $b N N$ vertices could make a contribution. Using the notation

$$
\Theta(p)=\frac{m-\not p}{2 m}=\frac{S^{-1}(p)}{2 m}
$$

where $S(p)$ is the propagator of the off-shell nucleon with four momentum $p$, scalar $\sigma N N$ vertex functions have the form

$$
\Lambda^{\sigma}\left(p, p^{\prime}\right)=g_{\sigma} \mathbf{1}-v_{\sigma}\left[\Theta(p)+\Theta\left(p^{\prime}\right)\right]
$$

and the pseudovector form of the $\pi N N$ vertex function is

$$
\Lambda^{\pi}\left(p, p^{\prime}\right)=g_{\pi}\left[\gamma^{5}-\Theta(p) \gamma^{5}-\gamma^{5} \Theta\left(p^{\prime}\right)\right]=\frac{g_{\pi}}{2 m} \phi \gamma^{5} .
$$

If the nucleons are on their positive energy mass shell, the action of the off-shell operator $\Theta$ on the nucleon spinor gives zero, so that only when the nucleon is off-shell will the $\Theta$ terms give a nonzero contribution, and in this case the scattering is sensitive not only to the $\sigma N N$ coupling strength $g_{\sigma}$, but also to the off-shell parameter $v_{\sigma}$, both of which are adjusted to fit the NN scattering data. Eq. (2.3) shows the well known equivalence of the pseudoscalar and pseudovector forms of the $\pi N N$ coupling for on-shell nucleons. There are similar off-shell terms for the vector couplings; for details see Ref. [18].

In 1997 [13] we discovered that the both the fits to the NN data and the three-body binding energy are very sensitive to the value of $v_{\sigma}$. Specifically, the value of $v_{\sigma}$ that gives the best fit to the scattering data (including the deuteron binding energy) also predicts the correct triton binding energy. This is a robust result that holds for all models studied (see Refs. [13] and [18]), and leads to the remarkable conclusion that CST OBE models predict the correct triton binding energy without three-body forces. This will be discussed in more detail in the following subsection.

\subsection{An equivalence theorem}

How can it be that CST models can predict the triton binding energy without three-body forces, whereas all other approaches require three body forces? The answer to this is fascinating; it gives us insight into the nature of three body forces and leads to a new equivalence theorem.

To illustrate what is involved, consider the cases shown in Fig. 2. Here the off-shell projection operator, $\Theta(p)$, cancels the neighboring propagator, $S(p)$, joining two neighboring points in the 

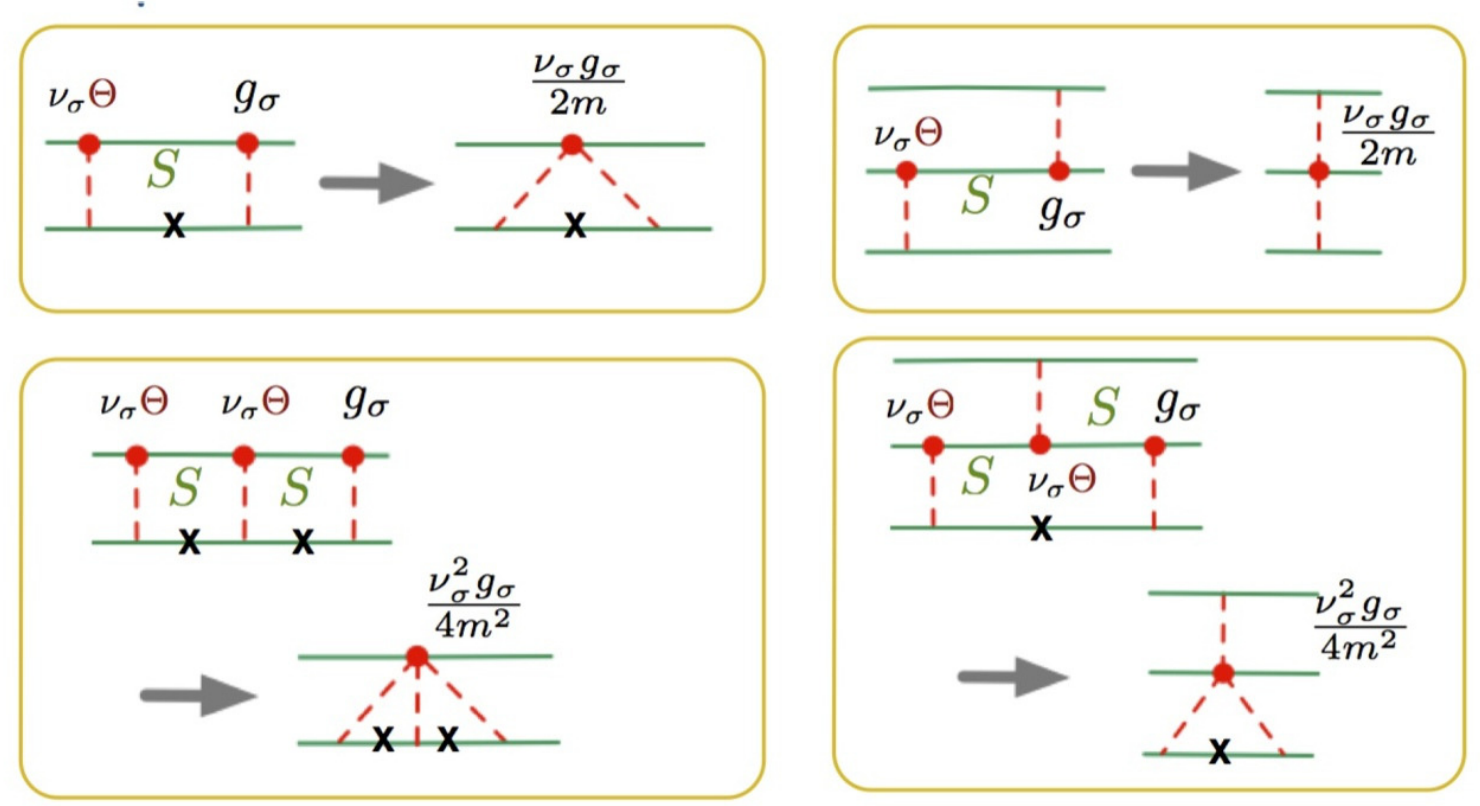

Figure 2: CST Feynman diagrams showing how off-shell couplings combine with off-shell nucleon propagators to give non-OBE type diagrams. Left panels show the collapse of box and double box diagrams into triangle and double triangle diagrams; right panels the collapse of iterated OBE diagrams into three body force diagrams. In all cases, $\Theta(p) S(p)=\frac{1}{2 m}$.
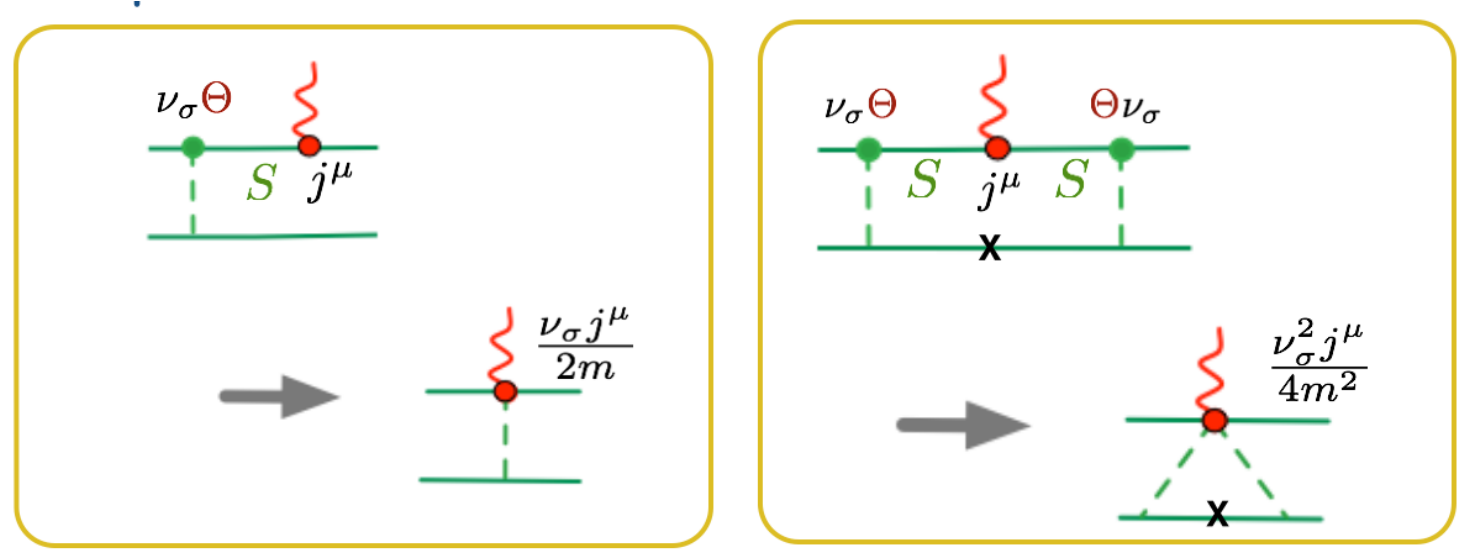

Figure 3: CST Feynman diagrams showing how off-shell couplings combine with off-shell nucleon propagators to give effective interaction currents.

Feynman diagram. In this way, triangle diagrams and three body force diagrams can emerge from an OBE model. In the same way, new interaction currents not found in the OBE model can emerge, as shown in Fig. 3. These cases illustrate a new equivalence theorem:

A CST OBE model with off-shell couplings (PictureA) is equivalent to another CST OBE model without off-shell couplings plus an infinite sum of specific non-OBE terms, many body forces, and non-OBE interaction currents, all with couplings determined by specific combinations of the OBE parameters (Picture B). 
So, with this equivalence theorem in mind, we might ask the question again: "Are there three-body forces?"

The answer clearly depends on whether or not we use Picture A or Picture B. Since I am using Picture A for these calculations, it is correct to insist that there are no three-body forces, even though it is possible to use the equivalence theorem to recast Picture A into Picture B. Picture B is useful primarily because it shows why methods that do not include these off-shell effects need three-body forces. Furthermore, is is important to realize that I could not actually use Picture B to do these calculations because it includes an infinite number of three-body force diagrams that I could not handle explicitly, and because the strength of these three-body force diagrams can only be determined from Picture A. The equivalence theorem, as it applies to the three body sector, is a particularly striking example of the general principal that has been known for a long time: three body forces do not "come from God" but depend on how we organize our theoretical calculations (Peter Sauer emphasized this in many of his talks).

I conclude by emphasizing a central point that this discussion of the equivalence of Pictures $\mathrm{A}$ and $\mathrm{B}$ that needs to be remembered - the strength of the three-body force terms in Picture $\mathrm{B}$ are uniquely predicted but the OBE couplings of Picture A. This powerful result suggests that the three-body force parameters are not really independent of the two body force, as many models suggest, and is subject to experimental tests. I expand on this point in the next section, where I compare $\chi \mathrm{EFT}$ to the CST.

\section{Comparison between $\chi$ EFT and the CST calculations}

In view of the success of the recent $\chi \mathrm{EFT}$ and CST calculations of the quadrupole moment, it is natural to compare the two and ask if they treat the physics differently. I will discuss how these two theories treat both the NN interaction and the two body current.

\subsection{The NN interaction}

In Fig. 4, diagrams describing the NN interaction in the two approaches are compared. The left panel selects diagrams up to NLO in $\chi$ EFT (this particular figure was extracted from Ref. [21]) and the right panel are contributions from pion and vector meson exchanges in Picture B of the CST OBE. The leading order $\chi$ EFT diagrams include a contact interaction and the one-pion exchange diagram. The one pion exchange diagram in both approaches is directly comparable. To compare the contact interaction, expand the heavy meson CST propagators in inverse powers of the meson mass squared

$$
\frac{1}{m_{v}^{2}-q^{2}} \simeq \frac{1}{m_{v}^{2}}+\frac{q^{2}}{m_{v}^{4}}+\cdots
$$

Then the first term in this expansion might reproduce the $\chi$ EFT contact interaction, provided its strength, $C_{0}$, satisfies the relation (here I ignore the spin and isospin operators so the comparisons are only symbolic)

$$
C_{0} \simeq \sum_{v} \frac{g_{v}^{2}}{m_{v}^{2}}
$$




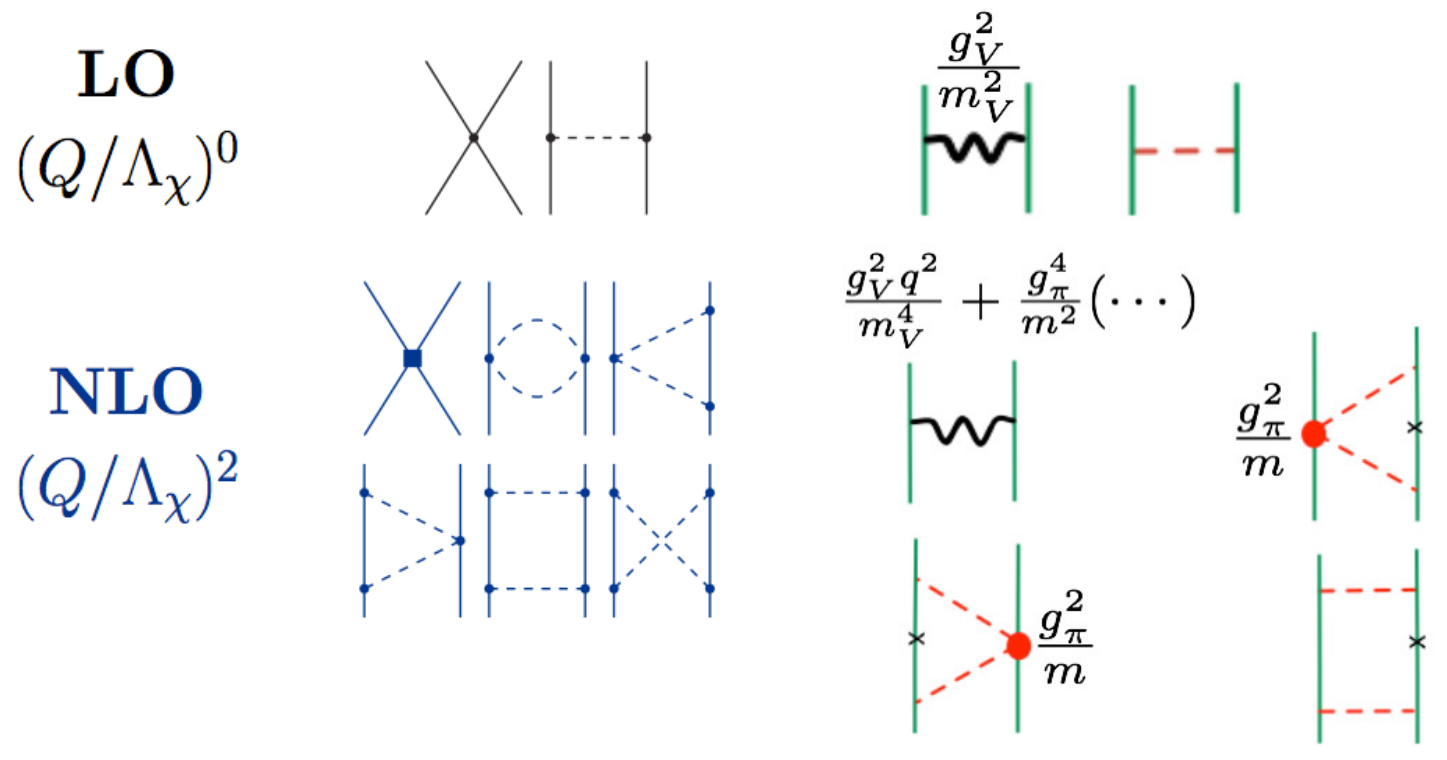

Figure 4: Left panel: $\chi$ EFT diagrams to NLO; right panel: parts of the pion and vector meson exchanges from CST OBE in Picture B. The mathematical expressions for the effective couplings shown in the right panel (not discussed in the text) are a start on a quantitative comparison between the two approaches. In these diagrams time flows from the bottom to the top.

This comparison provides a first relation of the parameters of the two approaches, providing a first test.

The comparison at NLO already becomes much more complicated. First, recall the CST cancellation theorem $[8,9,10]$ shows that, for isoscalar exchanges, the sum of the ladder and crossed ladder diagrams (without off-shell couplings) are well approximated by the spectator pole contribution from the box diagram alone. However, for isovector exchanges (which includes the pion) an additional bubble-like contribution must be added [9], which can be absorbed into the effective exchange of the heavy boson with the same spin and isospin (in practice these bubble contributions are distributed among more than one boson with different spins and isospins). This decomposition is illustrated in Fig. 5. The heavy boson exchanges of the CST OBE model are thus the sum of the contributions of physical bosons, plus contributions that remain after the cancellations between the box and crossed box contributions. (The success of the CST OBE models shows that, after the fact, this simplification works remarkably well.) The picture is further complicated when one sees that only the $q^{2}$ term in the expansion (3.1) will contribute at NLO - the leading term in the expansion of the heavy boson propagators already enters at LO.

Therefore the physics in the four non-triangle diagrams that arise in $\chi$ EFT at NLO is included

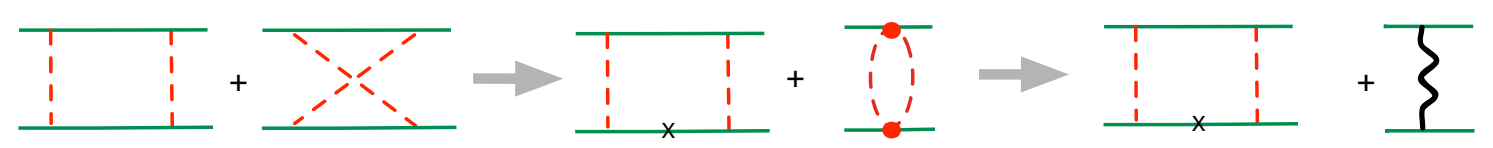

Figure 5: The Feynman 4th order ladder and crossed ladder diagrams is first expressed in terms of the single box diagram with one particle on-shell plus an irreducible bubble-type diagram, which is then absorbed into the heavy meson exchanges included in OBE models. 
in the CST box and effective meson exchange diagrams (without any off-shell couplings). This leaves only the triangle diagrams, and as we have already seen such contributions are generated (in Picture B) when off-shell couplings are added to the $b N N$ vertex functions, as discussed in the previous section and illustrated in Fig. 2. It is clear that all of the mechanisms in $\chi$ EFT (at least up to NLO) have counter parts in CST OBE models, and a detailed comparison is possible. Making this comparison quantitative is complicated by the fact that the $\chi$ EFT and CST theories define NN kernels (potentials) that are normalized differently and are designed to be used in different scattering equations, and that there are many more terms to consider at higher orders. Such a quantitative comparison is yet to be started, but if carried out might yield promising insights into the relative importance of the different mechanisms that give rise to the $\mathrm{NN}$ interaction.

\subsection{Two-body current}

The lowest orders for the two-body electromagnetic operators in $\chi$ EFT are shown in Fig. 6 taken from Ref. [7]. As is done in nonrelativistic theories, the charge and current operators are treated separately, and, to the order $Q^{3}$ shown, the only interaction currents involve a single pion. The CST, being relativistic, describes the electromagnetic interaction in terms of a single fourvector current, using the Feynman diagrams shown in Fig. 7.

Note that there is no analogue for the $\chi$ EFT charge operator (e) (the famous pion "in flight" term) in the CST; since Feynman diagrams are not time ordered, such diagrams are automatically
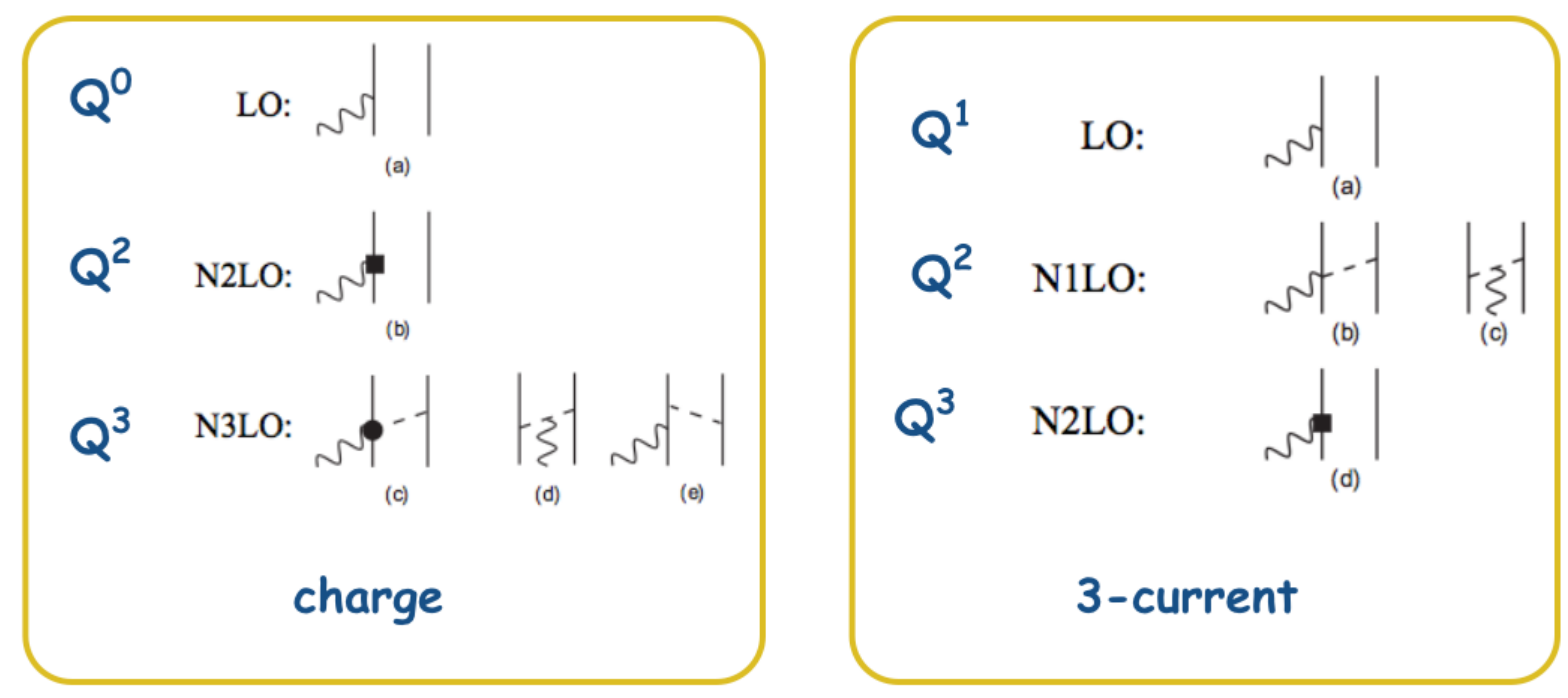

Figure 6: The charge operator (left panel) and the current operator (right panel) in the lowest three orders of $\chi$ EFT.

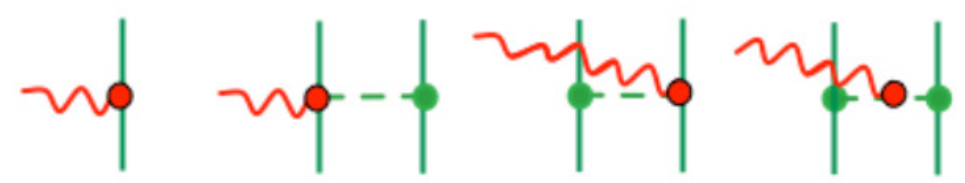

Figure 7: The irreducible four-current in CST OBE (Picture A). If the dashed line represents the sum of the exchange of all the mesons in the OBE model, these diagrams are a complete representation to the irreducible current; there are no more terms. 
reducible and their contributions included as part of the one pion exchange contributions from the wave functions. Even in the language of $\chi \mathrm{EFT}$ the contribution from these terms is largely removed by the renormalization process.

With the exception of the pion in-flight term, the comparison between the two theories at this order is more straightforward than it was for the NN interaction. It is significant that both theories use the free nucleon form factors as part of the one-nucleon current operator. In CST, in order to preserve current conservation exactly, the single nucleon current operator is modified by the strong form factor of the off-shell nucleon (this point is discussed in many references; for a recent discussion see Ref. [19]), but the use of the free form factor is at least consistent with the general philosophy of treating all of the hadrons as composite particles with structure. Use to the free nucleon form factor is less justified in $\chi \mathrm{EFT}$, where a fully consistent treatment would require calculation of the nucleon form factor using the same power counting used elsewhere in the calculation.

The comparison of the two approaches at higher order becomes more complex. There are many two-body interaction currents in the $\chi$ EFT expansion, some of which have the same structure as the interaction currents derivable in Picture B of the CST, one of which is shown in the left panel of Fig. 3 (note that this contribution must be added to the irreducible contribution shown in Fig. 7 before the comparison is made). Again, a detailed quantitative comparison has not yet been attempted and might yield significant insight into the importance of the various contributions.

\section{Origin of CST contributions to the quadrupole moment}

The major contributions to the quadrupole moment are summarized in Table 2. The leading term, expressed in terms of the usual S and D-state wave functions, $u$ and $w$, appears to be identical to the familiar nonrelativistic result

$$
Q_{\mathrm{NR}}=\frac{\sqrt{2}}{10} \int_{0}^{\infty} r^{2} d r\left\{u w-\frac{w^{2}}{\sqrt{8}}\right\}
$$

except that, in CST, the wave functions $u$ and $w$ satisfy different normalization conditions. The nonrelativistic and CST normalization conditions are

$$
\int_{0}^{\infty} k^{2} d k\left(u^{2}+w^{2}\right)= \begin{cases}1 & \text { nonrelativistic } \\ 1-\left\langle\frac{\partial V}{\partial m_{d}}\right\rangle-\int_{0}^{\infty} k^{2} d k\left(v_{t}^{2}+v_{s}^{2}\right)=1.023 & \text { CST (WJC-2). }\end{cases}
$$

If the leading term is evaluated using the nonrelativistic normalization condition, it is less that the experimental value $\left[Q_{d}=0.2859(6)\right]$ by $4.1 \%$; with the correct CST normalization it is less by only $1.8 \%$. The only remaining contributions that are significant at the level of $0.1 \%$ are relativistic corrections coming from the expansions of the of the relativistic kinematic factors in the many terms that contribute to the exact result (for details, see Ref. [6]), and these corrections bring the final result up to within $1 \%$ of the experimental value.

It is of interest to examine the origin of the normalization correction. As it turns out, the two P-state probabilities for model WJC-2 are very small, with a combined contribution much less than $0.1 \%$, so the normalization correction comes from the total energy derivative of the matrix element 


\begin{tabular}{lll}
\hline & error & physical origin and comments \\
\hline 1 & $-4.1 \%$ & $\begin{array}{l}\text { leading term with nonrelativistic normalization gives a result too small } \\
\text { (in line with previous calculations) }\end{array}$ \\
2 & $+2.3 \%$ & relativistic normalization correction \\
& & $-1.8 \%$ derivative of the strong nucleon form factor \\
& $+1.1 \%$ interaction current from off-shell particle \\
& & $+3.0 \%$ interaction current from mechanisms that force the on-shell particle off-shell \\
3 & $+1.0 \%$ & relativistic $k / m$ corrections to leading terms
\end{tabular}

Table 2: Origin of corrections to the quadrupole moment, expressed as a percentage "error" with respect to the experimental value, for model WJC-2 [6].

of the kernel, $V$. While its total size can be calculated explicitly from the models, detailed examination shows that its origin can be traced to contributions from the energy dependence of the strong nucleon form factors introduced to model the nucleon self energy (and to regularize the calculation), and contributions from the interactions currents that arise from the energy dependence of the off-shell projection operators $\Theta(p)$ (recall the discussion in Sec. 2.3). The size of the contributions from each of these sources is given in Table 2 .

While it turns out that the bulk of the correction comes from the normalization, I did not anticipate this in advance and it came as a surprise. The full calculation included many other contributions from the exchange currents and the P-states, but as it turns out, all of these are too small to be of importance. Some of these terms are important for model WJC-1, which has much larger P-states; in particular there is an interesting $w v_{t}$ interference term (where $v_{t}$ is the spin triplet P-state wave function) which is negligible for model WJC-2 but large and negative for WJC-1, accounting for the failure of this model to correctly predict the quadrupole moment.

\section{Discussion}

Both the CST OBE and $\chi \mathrm{EFT}$, with the right choice of NN parameters, are able to predict the quadrupole moment within $1 \%$ of its experimental value. However, both of these predictions show unwanted dependence on the models used to fit the NN data (for $\chi \mathrm{EFT}$, it is the cutoff, and for CST OBE it is the choice of model WJC-2 over WJC-1). Furthermore, at higher orders in the $\chi$ EFT expansion, new contact terms with unknown couplings enter, and these could be adjusted to fit the quadrupole moment, robbing $\chi \mathrm{EFT}$ of the ability to predict this quantity.

Comparing the two methods leads to a better understanding of the role of the off-shell CST OBE couplings, and to the conjecture that the $\chi$ EFT contact terms might be determined from the fewer OBE parameters, which would prove that CST OBE and $\chi$ EFT include exactly the same physics. If this should turn out to be true, it would give confidence that the truncation of the $\chi$ EFT series at the present order, or the approximations made in the use of the OBE mechanism in CST, are both sufficient.

I call attention to the recent review written by members of both the CST and $\chi$ EFT community [7]. This review concludes that the calculation of the deuteron form factors at high $Q^{2}$ (not dis- 
cussed in this talk) requires a fully relativistic theory. This is no surprise, since $\chi \mathrm{EFT}$ is based on a perturbation expansion which must fail at higher $Q^{2}$. However, at low $Q^{2}$ (recall that quadrupole moment is extracted from the computation of the quadrupole form factor, $G_{Q}$ at $Q^{2}=0$ ) both can be used, and it is gratifying that similar results can be obtained in the region where both are valid.

\section{References}

[1] F. Gross, J. W. Van Orden and K. Holinde, Phys. Rev. C 45, 2094 (1992).

[2] R. B. Wiringa, V. G. J. Stoks and R. Schiavilla, Phys. Rev. C 51, 38 (1995).

[3] R. Machleidt, Phys. Rev. C 63, 024001 (2001).

[4] Y. Huang and W. N. Polyzou, Phys. Rev. C 80, 025503 (2009).

[5] M. Piarulli, L. Girlanda, L. E. Marcucci, S. Pastore, R. Schiavilla and M. Viviani, Phys. Rev. C 87, 014006 (2013).

[6] F. Gross, Phys. Rev. C 91, no. 1, 014005 (2015) [arXiv:1411.7076 [nucl-th]].

[7] L. E. Marcucci et al., arXiv:1504.05063 [nucl-th].

[8] F. Gross, Phys. Rev. 186, 1448 (1969).

[9] F. Gross, Phys. Rev. C 26, 2203 (1982).

[10] F. Gross, New York, USA: Wiley (1993) 629 p

[11] F. Gross and D. O. Riska, Phys. Rev. C 36, 1928 (1987).

[12] J. W. Van Orden, N. Devine and F. Gross, Phys. Rev. Lett. 75, 4369 (1995).

[13] A. Stadler and F. Gross, Phys. Rev. Lett. 78, 26 (1997) [nucl-th/9607012].

[14] A. N. Kvinikhidze and B. Blankleider, Phys. Rev. C 56, 2973 (1997) [nucl-th/9706052].

[15] F. Gross, A. Stadler and M. T. Pena, Phys. Rev. C 69, 034007 (2004) [nucl-th/0311095].

[16] S. A. Pinto, A. Stadler and F. Gross, Phys. Rev. C 79, 054006 (2009) [arXiv:0901.4313 [nucl-th]].

[17] S. A. Pinto, A. Stadler and F. Gross, Phys. Rev. C 81, 014007 (2010) [arXiv:0911.1473 [nucl-th]].

[18] F. Gross and A. Stadler, Phys. Rev. C 78, 014005 (2008) [arXiv:0802.1552 [nucl-th]].

[19] F. Gross, Phys. Rev. C 89, no. 6, 064001 (2014) [arXiv:1404.1582 [nucl-th]].

[20] F. Gross, Phys. Rev. C 89, no. 6, 064002 (2014) [arXiv:1404.1584 [nucl-th]].

[21] R. Machleidt, published in NN and $3 N$ Interactions, L. Blokhintsev and I. Strakovsky, editors, Nova Science Publishers, Inc., New York, 2014 